\title{
Treatment of attention deficit in a patient with intractable daily seizures: a case report
}

\author{
Moody, Erika L* and Ivanhoe, Cindy B \\ Department of Physical Medicine and Rehabilitation, Baylor College of Medicine, Houston, Texas, USA
}

\begin{abstract}
Individuals with epilepsy often demonstrate a diminished capacity for sustained attention, whether from the underlying process or their medications. Some consider the use of stimulants such as Methylphenidate, controversial due to concerns they may lower convulsive threshold. This case report describes a patient with daily intractable seizures despite multiple anti-epileptic agents, with severe impairments in attention, cognition and communication. During an acute inpatient rehabilitation admission, this patient was treated with Methylphenidate and decreased anti-epileptic medication with clinically demonstrated improvements in attention, communication and command following. There were no adverse effects demonstrated in seizure frequency.
\end{abstract}

\section{Introduction}

Mechanisms responsible for the attention impairment in epileptics may be different from those involved in isolated AttentionDeficit-Hyperactivity Disorder (ADHD) [1]. Often a combination of anti-epileptic drugs (AEDs) used in combinations are required for intractable epilepsy, but this can also exacerbate drug-induced side effects such as slowed cognition and sedation [2]. Symptoms of inattention may improve in an individual with epilepsy through better seizure control, decreasing AED polytherapy or changing to AEDs with fewer behavioral or cognitive side effects [1]. Another treatment approach is the use of stimulants.

A controversial issue is the use of stimulants like Methylphenidate Hydrochloride (MPH), in individuals with epilepsy, as it is commonly believed to lower seizure threshold [3-6]. The electronic version of the Physician's Desk Reference 2014 reports that MPH "may lower convulsive threshold; $d / c$ if seizures occur [6]." Many other handbooks discourage the use of stimulants not only in individuals with active epilepsy but also in individuals, who are in remission and even in patients with epileptiform discharges on the electroencephalogram (EEG) alone $[4,7]$. There are variable incidences reported in the literature of the association of MPH and seizures. In one study, seizures occurred in two percent of the children in the stimulant-treated group (4 patients out of 205). However, in patients with coexistent ADHD and epileptiform changes on EEG, the risk may be as high as $20 \%$, whereas normal or nonepileptiform EEGs were strongly indicative of seizure risk less than one percent [3]. Additionally, there are reports which show that MPH can be used safely in individuals with well-controlled epilepsy $[3,4]$. There are only rare clinical cases in which seizure exacerbation has been reported with use of MPH but no randomized controlled trials that demonstrate an increased seizure frequency or severity in patients with epilepsy who are treated for ADHD with MPH $[1,8]$.

There is little data available documenting the safe use of MPH in patients with impaired attention and coexisting active seizures or EEG abnormalities $[4,5]$. The purpose of this case report is to describe the clinical changes observed in a patient with intractable seizures with the addition of MPH as well as a decrease in AED polypharmacy.
This patient had improvements in attention with no adverse effects on seizure frequency with MPH therapy.

\section{Case description}

A 21-yr-old male with epilepsy and daily intractable seizures since age 11 was admitted for acute inpatient rehabilitation. The patient had a history of craniotomy for grid placement complicated by subdural hematoma and a history of corpus callosotomy with a right frontal lobe resection with later development of meningitis. He also had placement of a vagal nerve stimulator (Figure 1). Prior brain imaging demonstrated encephalomalacia in the right frontal lobe and generalized parenchymal volume loss also involving the brainstem and cerebellum (Figure 2). EEG performed two months prior to admission revealed left-sided focal seizures with some multifocal generalized epileptiform discharges. Medications on admission included Clobazam, Levetiracetam and Divalproex Sodium. Given the persistence of daily seizures and secondary safety concerns, the patient was monitored with 24 hour constant visual observation. Prior to admission, the patient was diagnosed with a developmental intellectual disability and upon Neuropsychology evaluation, his IQ level was 40 . Several years prior, he was reportedly diagnosed with ADHD. Throughout the course of the physical examination, the patient was alert but demonstrated impairments with following simple 1-step commands, decreased attention described as "wandering and easily distractible," had prolonged response times and poor eye contact. He also demonstrated severe dysarthria and dyskinesia.

The patient was initiated on MPH during his inpatient rehabilitation,

Correspondence to: Erika Moody, Department of Physical Medicine and Rehabilitation, Baylor College of Medicine, 7200 Cambridge Street, Suite 10C, Houston, TX 77030, USA, Tel: 1-513-518-0739; Fax: 713-797-5982; E-mail: erika.moody@bcm.edu

Key words: seizures, epilepsy, methylphenidate, attention deficit disorder, cognition Received: January 15, 2016; Accepted: February 05, 2016; Published: February 08, 2016 


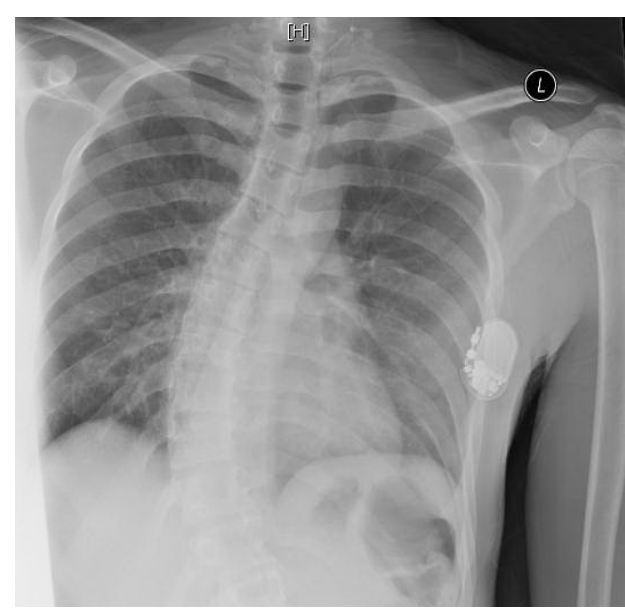

Figure 1. Vagal stimulator overlying the left lateral chest with the distal lead extending to the base of the left neck. Also noted is prominent dextroscoliosis of the lower thoracic spine.

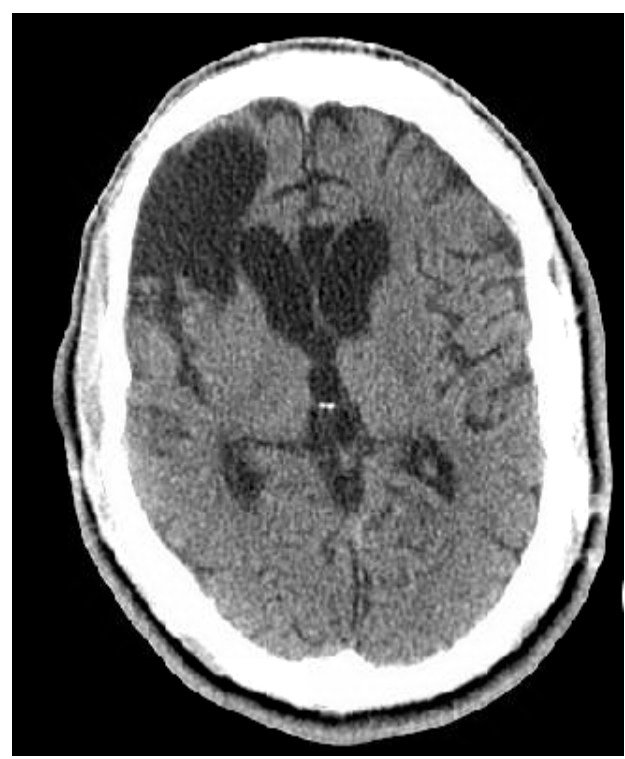

Figure 2. Head CT without contrast demonstrating right frontal operculum and corpus callosum encephalomalacia and generalized parenchymal volume loss.

which was titrated to $5 \mathrm{mg}$ twice daily. He was closely observed for adverse and therapeutic response over a 29 day period. He was weaned from $500 \mathrm{mg}$ twice daily dosing of Divalproex Sodium to $250 \mathrm{mg}$ twice daily. Clinically, he had improvement in seizure frequency during the admission with up to 72 hour seizure-free periods. He was unable to tolerate further reduction of Divalproex Sodium due to worsening of his mood and behavior as well as an increase in dyskinesia. Thus, he was continued on $250 \mathrm{mg}$ twice daily dosing. Improvements were demonstrated in language comprehension during simple conversation and the ability to follow simple 1-step commands in the context of an activity with minimal cues required. He responded more accurately to simple yes/no questions regarding his needs and wants, biographical information and environment. He correctly identified simple objects in a field of two, had increased eye contact and attention to environmental stimuli and was able to attend to target tasks for 5-10 minutes with minimal cues. The Neuropsychology reassessment described the patient as "less impulsive" and able to participate in picture vocabulary testing (PPVT-IIIB). He was able to accurately point to correct words at the age 4-5 level and exhibited functional attention to these tasks. The patients' parents reported that he was speaking with the best intelligibility since prior to his corpus callosotomy four years prior, with improvements demonstrated in attention, vocal quality, intelligibility and communication. At discharge the patient continued on Clobazam, Levetiracetam with decreased dose of Divalproex Sodium of $250 \mathrm{mg}$ twice daily in addition to MPH.

In summary, this patient exhibited improvements in attention, impulsivity, command following and communication ability, which will enhance quality of life for him and his family. In addition, the initiation of MPH demonstrated no adverse effects on his seizure disorder.

\section{Discussion}

Lowering of the seizure threshold with stimulants such as MPH is commonly considered to be a risk of therapy despite accumulating evidence that challenges this notion. Historically, neurostimulants such as D-amphetamines have been proven effective in the treatment of seizures $[2,9,10]$. These sympathomimetic agents stimulate the central and peripheral nervous system. Epileptic seizures have been reported to occur rarely with amphetamines but may occur after the first dosing [11]. Livingston et al., in a 1975 JAMA article, reported that amphetamines play a significant role in the treatment of the epileptic patient, particularly in diminishing or alleviating anticonvulsant druginduced drowsiness [10].

$\mathrm{MPH}$ is a safe, rapidly acting neurostimulant used effectively in individuals with $\mathrm{ADHD}$, that has been demonstrated to improve classroom behavior in children, reduce impulsivity and enhance attention [2]. MPH resembles amphetamine and acts as a substrate for the cellular monoamine transporter, especially the dopamine transporter and less so the norepinephrine transporter $[1,12]$. It occupies and blocks the dopamine and norepinephrine transporters, disinhibits D2 autoreceptors on presynaptic dopaminergic neurons and activates D1 receptors of postsynaptic neurons. Thus, MPH acts as an indirect catecholaminergic agonist, increasing synaptic concentrations of dopamine and norepinephrine [12]. MPH has no effect on neurotransmitters such as gamma-aminobutyric acid (GABA), glutamate and aspartic acid or sodium and calcium channels, which all have been associated with the pathophysiology of epilepsy. Additionally, there is no clear evidence in the literature to support that MPH has adverse interactions with AEDs [1]. Some literature reports that MPH may inhibit the metabolism of Coumadin and AEDs, specifically Phenobarbital, Diphenylhydantoin and Primidone which may require dosage reduction with concomitant use of MPH [11].

This report supports MPH as safe and feasible in individuals with refractory epilepsy and inattention. Additionally, this patient had a clinical decrease in seizure frequency with MPH. In a study by Gucuyener et al. MPH did not change seizure frequency from baseline and was safe and effective in children with ADHD and concomitant active seizures or EEG abnormalities [5]. Wroblewski et al. reported a decrease in seizure rate in children with post-head-injury epilepsy who received $\mathrm{MPH}$. In addition, $\mathrm{McBride}$ et al. reported no change in the epilepsy course over four years of follow-up in 20 children with epilepsy and three children with epileptiform findings, who received MPH treatment [1]. Moore et al. in a study with MPH in adults with intractable seizures, found that MPH did not significantly alter AED concentration (Valproic acid and Phenytoin) suggesting that previously reported interactions may not be clinically significant. These patients 
demonstrated significant improvements over a 3-month period in attention and concentration scores, memory scores and emotional well-being scores without significant alteration in seizure frequency [2]. Feldman et al. assessed the safety and efficacy of MPH in children with seizures and attention deficit with a double-blind medicationplacebo crossover study with MPH in ten children, age 6-10 years old, without seizures receiving a single antiepileptic drug (Carbamazepine, Phenytoin, Phenobarbital, Valproic acid). During the study period, no children had seizures or significant changes in epileptiform features or background activity on EEG and AED levels were without change [13].

In this particular patient, while there were certainly risks associated with medication changes, there were also issues related to his rehabilitation potential, quality of life and care needs. When considering management of his rehabilitation, it was felt that the potential gains of improving his attention were worth it. In the end, his function improved in meaningful ways.

\section{Conclusions}

Patients with a dual diagnosis of epilepsy and inattention may have improvements in attention, cognition, fatigue and overall improved quality of life without change in seizure frequency or severity by decreasing polypharmacy and the implementation of MPH. There is little evidence to support the perception that stimulants such as MPH increase seizure risk. This case report further supports prior studies, which challenge the assumption that stimulants lower the convulsive threshold. More studies are needed to further evaluate the safety of other neurostimulants for use in this population.

\section{References}

1. Kaufmann R, Goldberg-Stern H, Shuper A (2009) Attention-deficit disorders and epilepsy in childhood: incidence, causative relations and treatment possibilities. $J$ Child Neurol 24: 727-733.[Crossref]
2. Moore JL, McAuley JW, Long L, Bornstein R (2002) An Evaluation of the Effects of Methylphenidate on Outcomes in Adult Epilepsy Patients. Epilepsy Behav 3: 92-95. [Crossref]

3. Hemmer SA, Pasternak JF, Zecker SG, Trommer BL (2001) Stimulant therapy and seizure risk in children with ADHD. Pediatr Neurol 24: 99-102.[Crossref]

4. Aldenkamp AP, Arzimanoglou A, Reijs R, Van Mil S (2006) Optimizing therapy of seizures in children and adolescents with ADHD. Neurology 67: S49-51.[Crossref]

5. Gucuyener K, Erdemoglu AK, Senol S, Serdaroglu A, Soysal S, et al. (2003) Use of methylphenidate for attention-deficit hyperactivity disorder in patients with epilepsy or electroencephalographic abnormalities. J Child Neurol 18: 109-112.[Crossref]

6. PDR Network, LLC: Methylphenidate Hydrochloride/Methylphenidate Hydrochloride ER. 2014, 10/5/2014, http://www.pdr.net/drug-summary/methylphenidate-hydrochloride-methylphenidate-hydrocholide-er?druglabelid $=767$.

7. Gross-Tsur V, Manor O, van der Meere J, Joseph A, Shalev RS (1997) Epilepsy and attention deficit hyperactivity disorder: Is methylphenidate safe and effective? $J$ Pediatr 130: 40-44. [Crossref]

8. Fosi T, Lax-Pericall MT, Scott RC, Neville BG, Aylett SE (2013) Methylphenidate treatment of attention deficit hyperactivity disorder in young people with learning disability and difficult-to-treat epilepsy: evidence of clinical benefit. Epilepsia 54: 2071-2081. [Crossref]

9. Livingston S, Kajdi L, Bridge EM (1948) The use of benzedrine and dexedrine sulfate in the treatment of epilepsy. J Pediatr 32: 490-494.[Crossref]

10. Livingston S, Pauli LL (1975) Dextroamphetamine for epilepsy. JAMA 233: 278-279. [Crossref]

11. Zagnoni PG, Albano C (2002) Psychostimulants and epilepsy. Epilepsia 43: 28-31. [Crossref]

12. Spiller HA, Hays HL, Aleguas A (2013) Overdose of drugs for attention-deficit hyperactivity disorder: clinical presentation, mechanisms of toxicity, and management. CNS Drugs 27: 531-543. [Crossref]

13. Feldman H, Crumrine P, Handen BL, Alvin R, Teodori J (1989) Methylphenidate in children with seizures and attention-deficit disorder. Am J Dis Child 143: 1081-1086. [Crossref]

Copyright: (C2016 Moody. This is an open-access article distributed under the terms of the Creative Commons Attribution License, which permits unrestricted use, distribution, and reproduction in any medium, provided the original author and source are credited. 